Corps et culture

Numéro 3 | 1998

Sport et lien social

\title{
Corps et lien social
}

\section{Mahmoud Miliani}

\section{(2) OpenEdition}

Journals

Édition électronique

URL : http://journals.openedition.org/corpsetculture/759

DOI : 10.4000/corpsetculture.759

ISSN : $1777-5337$

\section{Éditeur}

Association Corps et Culture

Édition imprimée

Date de publication : 1 juin 1998

ISSN : 1268-5631

Référence électronique

Mahmoud Miliani, «Corps et lien social », Corps et culture [En ligne], Numéro 3 | 1998, mis en ligne le 04 mai 2007, consulté le 23 septembre 2020. URL : http://journals.openedition.org/corpsetculture/ 759 ; DOI : https://doi.org/10.4000/corpsetculture.759

Ce document a été généré automatiquement le 23 septembre 2020

(c) tous droits réservés 


\title{
Corps et lien social
}

\author{
Mahmoud Miliani
}

1 Nos premiers échanges sur le thème du lien social ont eu lieu quelques semaines avant les grèves nationales qui survinrent à la suite de l'annonce du projet gouvernemental de restructuration ultra-libérale des services publics. Localement, à la Faculté des Sciences du Sport, les étudiants étaient en grève et protestaient contre les conditions d'accueil et de travail; deux projets de politique de formation et d'administration s'affrontaient ; la situation était confuse ; le traditionnel clivage politique ne permettait en rien de distinguer les différents acteurs des deux camps.

2 Le présent numéro n'a pas pour objet d'analyser les mouvements sociaux de cette période. Le dire c'est une manière de réactiver, si besoin était, une lecture critique dont l'objet serait, au moins, d'interpréter le travail d'euphémisation, de rationalisation, de dénégation propre au discours scientifique ou qui en emprunte l'allure. C'est aussi une manière de rappeler l'impensé sur lequel se constitue un champ, une institution, un laboratoire, un groupe. Quel sens accorder à cet avant-propos? Moment d'élucidation ou retour du refoulé? Au lecteur de juger.

Le mouvement social de l'automne 1995

3 Une fois au pouvoir sur la base d'un programme-remède à « la fracture sociale ", les hommes politiques, soutenus par le système médiatique, des experts en économie, certains intellectuels ralliés et enchantés par le discours sur la fracture, font de nouveau preuve de cynisme. Ils comptaient sur la léthargie du corps social, l'absence de mémoire, la démission des clercs pour continuer leur travail de domination. Aux uns incombaient le rappel des impératifs économiques, l'appel au sens des responsabilités, la culpabilisation des « privilégiés » de la fonction publique, la mise en place, par voie médiatique, d'une pédagogie de la soumission, aux autres, les topos lénifiants sur « la crise » ou « la perte du lien social », « la nouvelle pauvreté », « les exclus », etc., et les téléthons en tout genre qui donnent la fausse idée d'un communautarisme retrouvé, faisant oublier le temps d'une émission les souffrances endurées par des millions de précaires, de chômeurs, de "sans» quelque chose (abri, papiers, etc.). C'est le supplément d'âme ou d'idéologie de la pitié dont a besoin le libéralisme qui trouve en cette période le champ libre à sa politique de libre-échange ${ }^{1}$. 
4 En effet, les avancées de la mondialisation sont à présents indéniables ${ }^{2}$. Aussi, faut-il rendre hommage à Jean Chesnaux d'avoir eu la perspicacité, à l'encontre des intellectuels enchantés par le nouvel état du monde et autre âge de la démocratie, d'analyser les effets ravageurs de la modernité. Le sort des Etats-nations, montrait-t-il, se décide dans les grandes Bourses du monde. De la City de Londres, en passant par Wall Street, Tokyo, Bahrein et ses pétrodollars, les considérations «globales » tendent à effacer le «local » et à lui dicter le rythme et les exigences de l'économie mondiale. Pour l'auteur, la "planète câblée " n'est pas une métaphore mais une réalité. Du fait de «l'interconnexion des ordinateurs, le marché financier mondial fonctionne vingtquatre heures sur vingt-quatre en ubiquité instantanée, et la même interdépendance mondialisée caractérise toute la vie économique de la modernité $»^{3}$. La mondialisation ne serait pas une nouvelle étape de croissance du système capitaliste. Ce ne sont pas les critères de changement de taille et de structure (Firmes transnationales) qui rendraient intelligible le phénomène. La mondialisation présente une "nouveauté radicale, intrinsèque " et des conséquences sociales et politiques. La nouveauté c'est un "renversement de priorités». Ce ne sont plus les orientations nationales ou les rythmes de l'activité locale qui déterminent, comme dans le capitalisme classique, la politique économique mais les perspectives et les contraintes du champ économique mondial. Les nouveautés ce sont, en outre, les traits suivants : le « hors-sol » qui caractérise une économie à haut rendement et coûts faibles de production et dont les conséquences sont la disparition de secteurs entiers de l'économie rurale ; les échanges des « marchés électroniques » où la spéculation est la loi et où les transactions représentent des opérations purement financières dissociées des activités de production c'est-à-dire sans valeur concrète; l'interconnexion de tous les secteurs de l'économie mondiale avec « des effets de chaîne» que les Etats (qui ne sont plus souverains) sont incapables de maîtriser Les conséquences de la mondialisation sont premièrement la société duale, la nouvelle pauvreté. Deuxièmement, l'effacement du «local» au profit du «global»; autrement dit, la disparition des «arts de vivre locaux, [des] solidarités locales sur lesquelles se fonde l'identité collective des peuples et des nations $»^{4}$. Troisièmement, le changement de la fonction de l'Etat national d'un côté asservi aux impératifs de l'économie mondialisée et d'un autre côté se faisant gestionnaire du chômage, de la pauvreté, de la délinquance 5 .

5 En cet automne 95, les technocrates de l'ENA alliés à une philosophie sociale qui fait du marché l'horizon indépassable de l'humanité, ne pouvaient pas imaginer que le bon peuple, sage, à qui il faut constamment expliquer les raisons économiques puisse se révolter contre les lois de l'économie. Ils ne se doutaient pas non plus qu'il n'y ait pas que des experts, des conseillers du prince dans le champ scientifique. Qu'il existe dans la société en deçà de la "programmation guidage» de la population et du conditionnement des esprits une énergie sociale insoupçonnée et des intellectuels prêts à prendre leur responsabilité.

6 Parmi les grévistes de la gare de Lyon, Pierre Bourdieu s'en prend à «cette noblesse d'Etat, qui prêche le dépérissement de l'Etat et le règne sans partage du marché et du consommateur, substitut commercial du citoyen, [et qui] a fait main basse sur l'Etat, [en faisant] du bien public un bien privé [ ... ] Ce qui est en jeu, poursuit-il, c'est la conquête de la démocratie contre la technocratie : il faut en finir avec la tyrannie des " experts ", style Banque mondiale ou FMI, qui imposent sans discussion les verdicts du nouveau Léviathan, les marchés financiers ", et qui n'entendent pas négocier, mais " 
expliquer " il faut rompre avec la nouvelle foi en l'inévitabilité historique que professent les théoriciens du libéralisme; il faut inventer les nouvelles formes d'un travail politique collectif capable de prendre acte des nécessités, économiques notamment [ ... ], mais pour les combattre et, le cas échéant, les neutraliser.» (Libération, 14.12.95)

7 Alors que Pierre Bourdieu parmi les cheminots [donc contre « les intellectuels de cour et d'écran $»^{6}$ réinvente une figure de l'engagement ${ }^{7}$, d'autres intellectuels prennent la parole, agissent non pas en s'attaquant à l'hégémonie du marché et de la pensée managériale mais en dévoilant l'emprise de la circulation et du mouvement forcé imposés quotidiennement aux citoyens. L'arrêt, la grève livrent en quelque sorte les fonctions cachées des transports en commun, des flux effrénés sur la route et leur fonction dissolvante du lien social.

Edgar Morin écrit: «En effet, l'électrochoc qui a soudainement immobilisé tous les transports a réveillé un pays qui s'était somnambulisé dans le métro-boulot-dodo. Le métro suspendu, le boulot chahuté et le dodo raccourci ont soudain suscité des proliférations de débrouillardises, ingéniosités et solidarités. Le réveil généralisé et multiple de la solidarité, entre travailleurs d'un même centre ou dépôt, entre ces travailleurs; leurs familles, leurs amis et voisins, et la naissance de communications et entraides entre voisins d'habitation ou de travail montrent que la paralysie de la grève a provoqué comme une régénération spontanée du tissu social et a fait retrouver la santé psychique minimale qui comporte l'ouverture à autrui. Du coup, nous pouvons dire à quel point dans la situation dite normale il y avait la "déliaison" généralisée, l'isolement des individus, la raréfaction des communications affectives ... " (Libération 19-12.95). Presque au même moment Jean Baudrillard observe, à sa façon, la reconquête par les citoyens de l'espace public et la redécouverte de la temporalité propre au corps. Toutes choses fortes de conséquences sociales et politiques. « Marcher, marcher, écritil, ç'aura été la grande révélation de ce mouvement. Beaucoup plus que les manifestations traditionnelles. Car le point crucial de la fracture sociale, c'est justement la circulation. La seule circulation dans cette société, c'est celle des élites et des réseaux, celle de l'argent et de l'information en temps réel. C'est contre cela que les gens marchent. Ils marchent dans le temps différé de l'espace, contre le temps réel des réseaux dans le temps physique des parcours, contre la circulation effrénée des flux. C'est une contestation originale et directe de la norme même de cette société.» (Libération, 18.12.95)

9 Je ne résiste pas ici au plaisir de rappeler combien ces observations sont redevables aux travaux de Paul Virilio et combien certaines de ses analyses sont pertinentes non seulement pour ce qui concerne les événements de l'automne 95 mais aussi pour notre rapport quotidien au mouvement, au corps. Souvenons nous des déclarations et injonctions du pouvoir par l'intermédiaire du Premier Ministre Alain Juppé. Avant toute discussion avec les grévistes il exigeait que l'on «rétablisse la circulation ». Car l'enjeu est bien là. Pour le pouvoir, la circulation c'est l'ordre; l'embouteillage (Cf. les récentes actions des chauffeurs de camions), le débrayage, la grève, surtout les rassemblements sont menaçants. A la rigueur le pouvoir préfère un défilé de manifestants qu'avoir affaire à une occupation. Le mot du pouvoir est d'abord « circulez!». Hier, cet ordre s'était appliqué aux squatters en tout genre, aux Maliens (sans abri) qui campèrent à Vincennes, aux «sans papiers » de l'église Saint-Denis à Paris; aujourd'hui aux chômeurs qui occupent les ASSEDIC, ANPE ou d'autres 
administrations, services ou institutions de l'Etat; de tout temps, note Virilio, la police a toujours obligé les marginaux, les mendiants à déambuler. Dans cette analyse du rapport à l'espace et au corps en mouvement le pouvoir apparaît moins une séquestration, un enfermement qu'une "dictature du mouvement $»^{8}$.

10 On comprend mieux la redécouverte d'autrui, les multiples solidarités, le vécu du rythme singulier du corps pendant la grève. Car habituellement, la ville n'est pas un lieu d'habitation et d'échanges sociaux et culturels; l'espace urbain est surtout un entrecroisement de voies de communication, un espace de circulation de plus en plus rapide ; les transports en commun ou prives ne sont rien qu'un moyen de déplacements en masse de corps-objets, d'individus atomisés.

11 La grève de l'automne 95 nous aura appris les conséquences négatives d'une vie sociale réduite à de la circulation. Il n'est pas inutile, par ailleurs, de mentionner que la tactique du pouvoir se traduit par l'obligation au mouvement. C'est une manière d'affronter l'un des impensés d'une éducation par le mouvement.

12 Pendant le temps social de la contestation, pendant cette période de l'entredeux où tout est possible, à la Faculté des Sciences du Sport de Montpellier il se passait beaucoup de choses. Seulement cela se passait à l'intérieur des murs de l'organisation. Ici, point de mouvement social mais un mouvement brownien qui agite les fantasmes et les membres de l'institution. Refermée sur elle-même, celle-ci fonctionnait (et fonctionne encore) sur le mode schizophrénique. La compréhension de cette situation locale relève moins d'une approche dans les termes d'une sociologie des organisations que d'une interprétation clinique des comportements déviants encouragés par l'isolement de l'institution.

Psychopathologie du lien à la faculté des sciences du sport de Montpellier

13 En 1995, un nouveau Doyen est nommé. Pour mieux traduire la dimension réelle et imaginaire de l'institution, il vaudrait mieux dire que l'ancien Doyen est renversé et, à travers lui, le (ou les) père(s) fondateur(s) de "la maison »". En cette rentrée universitaire qui voit multiplié par 4 le nombre des étudiants inscrits, avec tous les changements d'habitudes et les pertes de profits occultes que cela impose aux personnels hostiles à «la nouvelle politique », les débats s'enlisaient autour des questions de démocratisation, de quantité/qualité et de deux projets antagonistes en termes infrastructurels et de politique de(s) formation(s) (au singulier pour les uns, au pluriel pour d'autres). Mon propos n'est pas de reconstituer à la manière d'un détective de l'histoire le déroulement des faits ni de me placer au-dessus de la mêlée généralisée (où les coups bas sont la règle); il n'est pas question non plus d'exhiber, qui caractérise le jeu institutionnel; comme cela se fait en ce genre d'occasion, les armes de l'analyse institutionnelle et de m'en tenir quitte par l'analyse des implications; je voudrais seulement pointer quelques phénomènes (mé)connus et déniés par les agents dans la crise du lien institutionnel.

La polarisation du champ

14 Les bavardages de couloir plus ou moins courtois, les explications (au double sens) et empoignades verbales (quasi physiques) dans les réunions dites de concertation, la peur panique manifestée par certains agents, des accidents somatiques aussi, expriment à quel point «la nouvelle politique» en tant qu'elle est porteuse d'un nouveau rapport au corps et à l'esprit de corps met en danger des identités construites sur d'anciennes adhérences institutionnelles et des intérêts matériels (non négligeables) et symboliques. La lutte révèle les modes de structuration des groupes en 
opposition, et des types d'individus. Deux types de groupalité s'affrontent. L'enjeu en est leur inscription/imposition comme matrice, structure de base de l'institution. Il s'agit d'un groupe, à l'origine de cette institution, fondé sur une sociabilité syncrétique et d'un autre groupe constitué sur la base d'une sociabilité par interaction et dont le but est de chercher à réactiver le processus d'institutionnalisation à son bénéfice. La première définit un état d'indifférenciation dans lequel il est impossible aux individus de discriminer le moi et le non-moi, le corps individuel et le corps groupal, le je et le nous. Ce niveau de sociabilité est paradoxalement une non-relation. Par ailleurs, la nondiscrimination des individus entre-eux favorise la clôture de la pensée sur les thèmes de l'égalitaire et de l'identitaire (l'identique); la défense contre les angoisses paranoïdes en faisant bloc contre l'extérieur ; la défense des intérêts matériels et autres gratifications substantielles justifiés pour service rendu à la corporation. Le deuxième type de relation s'établit sur un accord rationnel en vue de civiliser les moeurs archaïques et de moderniser l'institution. Ce n'est pas que ce regroupement est le symbole d'une interaction démocratique; comme tous les groupes, lui aussi a son arrière-fond syncrétique et sectaire. La différence se situe plutôt dans la modalité dominante et dans le mode de reproduction ou de structuration. D'un côté le syncrétisme, de l'autre l'interaction; d'un côté l'homogénéité et le développement parthénogénétique, de l'autre l'individualisme dénié, l'association/coalition paradoxale d'ex-gauchistes avec une droite conservatrice et même avec un lepéniste déguisé en adversaire de la corruption. La polarisation groupiste de l'espace n'épuise pas la description des relations institutionnelles. Il existe quelques individus isolés, bien identifiés par les deux camps et étiquetés "atomes libres». Ce ne sont pas des girouettes. Car comme chacun sait, ce ne sont pas eux qui changent mais la direction du vent. L'espace n'est donc pas bipolaire mais comprend des individus incertains, sans parti ni parti pris. Leur mérite est d'ajouter du bruit, du désordre. Ils sont fiers de leur apport à la complication. Mais surtout ravis qu'aucun groupe ne peut les contenir Grâce à eux l'institution s'enrichit d'une sociabilité sans frontières. Placés entre les tenants du syncrétisme et ceux qui investissent dans les (inter)actions, les individus incertains forment sans le savoir le groupe des incontinents.

Pour l'instant on peut dire que la crise a pour origine la destruction d'un monde ancien avec ses liens spécifiques, sa chefferie de terroir, son mode de reproduction du corps et de l'esprit de corps, son principe de gestion occulte de l'institution; le remplacement de ce monde par une politique de transparence qui trouve aujourd'hui ses limites dans la froideur bureaucratique et le jeu d'apparence démocratique; le brouillage des frontières politiques et idéologiques; le tout dans une ambiance guerrière de meurtre $d u$ père fondateur et une éclosion de prétention à la fondation ${ }^{10}$.

L'hypertrophie de la mémoire

16 Habituellement les institutions souffrent d'un refoulement massif du passé. Les relations sociales apportent chaque jour leur part de sédimentation, de déni et de dénégation. La vie professionnelle se déroule tant bien que mal à travers des confrontations plus ou moins réglées, des fanfaronnades, des représentations théâtrales. Quand le risque est grand les acteurs développent un art de sauver la face (E. Goffman) par des comportements d'évitement, des faire-semblant, des simulacres d'indifférences, etc., autant de stratégies de maintien du cadre des interactions entre des périodes de crise, de retour cyclique du refoulé. Ce fonctionnement là serait le modèle de la normalité institutionnelle, puisque en dehors des accès de fièvre et des 
bronchites aiguës, l'institution poursuit ses finalités de formation, de recherche, d'éducation, de soins, etc.

17 A la Faculté des Sciences du Sport rien de tout cela. La crise est endémique. La production/reproduction de la réalité ne se fait pas sur fond d'oubli ou de majorité silencieuse. L'institution est malade d'une hypertrophie de la mémoire. Les réunions dites de concertation, les assemblées plénières et diverses autres modalités de rencontres pour préparer des avant-projets, réfléchir sur le présent et l'avenir, tirer des leçons de la dernière esquisse d'un projet fumeux qui ne verra pas le jour, tous ces moments institués ont été l'occasion d'une expression pathologique de la mémoire. Le phénomène en question consiste dans le rappel incessant par quelques gardiens $d u$ patrimoine collectif des souvenirs-écrans, des événements passés et ressassés à l'origine de «la crise », «du ras-le-bol», «du malaise », «du dysfonctionnement», « de l'insatisfaction ", etc., de tous ou d'une partie du personnel enseignant et administratif. Lors de ses séances dites de travail, où l'on se plaint que «cela n'avance pas » et que "de toute façon cela ne sert à rien", les représentants de la mémoire paranoïaque de chaque camp figent la discussion et le temps par un discours délirant, structuré par une série de plaintes, d'insultes, de vociférations haineuses, d'accusations en ping-pong et aussi par des éléments projectifs, de recherche à l'infini des responsables, de la cause du mal, etc.

Dans sa répétition maladive, cette théâtralité a fait pourtant évoluer le lien institutionnel. Aujourd'hui on ne vient plus évacuer «sa merde» ou se libérer de tensions internes d'origine institutionnelle, les représentants du passé traumatique sont fatigués par plusieurs crises intestines. Ils viennent épisodiquement nous rappeler les hallucinations rétrospectives et partagées dans des rôles pétrifiés ${ }^{11}$.

Les thanatophores

19 L'anomie suscite d'autres vocations. Il s'agit, précisons-le, d'un contexte de faillite de l'ancien dispositif organisationnel fondé sur un pacte tribal et sectaire et de remise en question des fonctionnements psychiques protégés et garantis par les complicités communautaires. Dans ce contexte, certains sujets se sont trouvés brutalement face à leurs manques, à leur vide intérieur jusque-là comblé par l'illusion groupale d'appartenance à l'Université. Face à la transformation, restructuration exigées par l'afflux de "nouveaux » étudiants, ils ont vécu d'abord avec angoisse la perte des étayages anciens ; puis prenant conscience de la lâcheté des membres de la Famille qui n'est plus Sainte, sentant «qu'on les traite comme de la merde », "qu'on les prend pour des cons ", ils se sont faits une nouvelle spécialité : porteurs de la négativité de l'institution. Ils apparaissent comme les mutants d'un moment aigu, catastrophique, dans le cours de l'anomie institutionnelle. Ce sont des thanatophores. Aujourd'hui abandonnés, «laissés pour-compte », n'ayant pas eu leur part de gâteau ou quelques miettes du festin institutionnel, enfin convaincu de leur faillite, ils ont su se recycler à temps pour porter la destructivité à chaque réunion où il était question d'élaborer, construire, penser ${ }^{12}$.

20 A ce jour, il existe deux types-idéaux de thanatophores. Les brailleurs, râleurs, destructeurs, hystériques. Ils portent l'agitation et s'agitent dans le style du bébête show. Ils faisaient rire. Mais voilà, cela fait deux ans qu'ils rejouent la même scène, avec la même émotion. Ils étaient certainement authentiques. Avec la momification de leur rôle, aujourd'hui, ils sont tragi-comiques. Ils sont agressifs mais pas méchants. Le deuxième type est plus dangereux. Il est silencieux, homme de marbre, porteur de 
haine froide. Il s'exprime rarement mais à son contact d'autres se chargent de déverser leur colère pour dire ce qui ne marche pas et bloquer ce qui risque de marcher. Si le premier type est violent mais seulement en gesticulations, le deuxième type qui est le vrai thanatophore est un tueur froid, un pervers qui trouve à jouir de la crise endémique et de laquelle, comme un malin génie, il s'alimente secrètement ${ }^{13}$.

Chronique d'un lien groupal

21 Octobre 1997, c'est la foule dans «Corps et Culture ». Vingt personnes. Tout le monde ne se connait pas. Le laboratoire agrège de nouvelles ressources intellectuelles. Par quelle voie ? Mystère. On en parle peu autour de nous. Yves Le Pogam, avec la finesse qu'on lui reconnaît, remercie tout le monde et explique, dans un discours spontané d'ouverture, notre présence par une attraction mystérieuse qui trouve son principe dans des affinités électives. A l'entendre, les relations enchantées d'hier, entre les 4 à 6 personnes qui maintenaient une activité de laboratoire, avaient malgré le nombre toutes les chances de continuer de soutenir le travail de réflexion collective. Mais l'enchantement fut de courte durée. La discussion s'est engagée sur la constitution du nouveau bureau, sur les statuts non conformes du laboratoire, sur «la nécessaire séparation entre l'Association et le laboratoire " (sic), sur la nécessité de réunir selon une périodicité légale les membres élus, sur les modalités du vote, sur les règles à suivre en cas de ...., sur etc., etc. Rires naïfs de certains (ceux qui ne se doutent de rien), rires gênés d'autres (eux ils savaient ou pressentaient), et enfin rires sérieux de ceux qui étaient attachés au bureau parce qu'instrument de régulation et incarnation de la volonté collective. Ceux-là tenaient cette croyance de l'expérience récente d'une OPA faite par quelques individus sur une revue dans laquelle ils n'avaient pas mesuré (au double sens) leur investissement. Ce jour d'octobre 1997, «Corps et Culture » entre dans la modernité par hybridation. Le bureau est transplanté dans sa structure domestique.

22 La référence à " l'ici et maintenant » a simple valeur métaphorique pour pointer une évolution. En réalité, depuis quelques mois le mouvement est irrésistible. La séance d'octobre 1997 n'est qu'un moment de constitution. Ce qui s'y est passé précipite sans doute de l'institution. La taille actuelle du groupe, l'éthique de la raison de certains membres, l'expérience d'autres lieux peu démocratiques régis par l'esprit de famille et l'autorité patrimoniale expliquent la propension à penser qu'un certain degré de bureaucratisation et de rationalisation est source de liberté ${ }^{14}$. Certes, est-ce une anticipation intelligente et légitime contre le détournement, toujours possible quelles que soient les relations de confiance internes au groupe, du laboratoire au service d'intérêts individuels et contre les contraintes des relations proximales potentiellement porteuses de violence méconnue. En revanche, je suis enclin à penser que la rationalisation ne connaît pas de limites. Mon hypothèse (tragique) est qu'elle affectera le corps et la culture du groupe et transformera ses «intérêts de connaissance » en intérêts rationalisés. Je ferai donc quelques observations pour anticiper les anticipations.

1) Le passage à la socialité secondaire ne doit pas se faire sur l'effacement de la socialité primaire. Ce rappel n'est en rien nostalgique d'un passé fantasmé; non plus animé par le mythe d'une communauté d'origine. C'est un impératif éthique selon lequel les relations entre les personnes doivent prendre le pas sur les relations entre les fonctions. De même la socialité secondaire, indispensable à la vie d'un laboratoire forcément sous le primat de "l'économie des biens ", doit être étayée sur «l'économie 
des hommes » (j'emprunte ces expressions à Louis Vincent Thomas) propre à la socialité primaire. Ce principe admis, on aura ainsi quelque chance d'établir des communications sur le mode du don et non pas sur celui de l'échange, et surtout, d'éviter de faire du don de parole une circulation de mots. Pour convaincre, il suffit de penser aux divers registres récents qui s'imposent imperceptiblement comme normes de communication. Alors que dans le groupe primaire le don de parole condensait la fonction de connaissance et celle de création du lien, l'échange tend à présent à chosifier la parole. Il y a celui qui ne donne rien mais prend à la dérobade ; celui qui reçoit les mots et les renvoie en grimaçant ; celui toujours prompt à prendre au vol la parole des autres pour l'approfondir, la vernir, à moins de la ternir ; l'inflationniste, lui, ne donne pas, il dépense, gaspille les mots, fait de la surenchère. Dans ce marché de la parole, les valeurs individuelles de signe et d'usage tendent à faire oublier la valeur de lien.

Que l'on comprenne. Il n'est pas question de faire de la morale ni de faire passer en contrebande une vision idyllique et pacifiée de la communication. Le don implique toujours l'obligation de rendre. L'intérêt est inscrit dans les actes les plus désintéressés. Le monde humain est trop humain. Le calcul voisine avec l'agapè, l'égoïsme avec la générosité. Mais il s'agit de ne pas dénier, rationaliser, voire transfigurer cette réalité. Surtout, il s'agit de construire un monde contre l'intérêt et non de désirer une socialité sans l'intérêt, ce qui paraîtrait à nos yeux d'humains comme un monde sans intérêt ${ }^{15}$.

2) On ne peut pas parler d'un «effet bureau» dans le laboratoire. Cependant, il vaut mieux en parler même en termes de fiction. Car comme chacun sait, la fiction finit par être «bien fondée ». Je pense à ces menus tâches perçues bénévoles parce que " emmerdantes", effectuées en toute simplicité, en toute modestie par celui qui se signale aux autres en se faisant oublier. Posture corporelle et mentale du serviteur de la revue ou du laboratoire. C'est quelqu'un qui n'a pas une fonction attribuée encore moins rétribuée (c'est impensable). Un être (encore fictif, je l'espère) qui fait preuve sans ostentation du don de son temps. Avec le temps et sans rien demander, il deviendra indispensable au bon fonctionnement de la structure. Le voilà permanent. Les tâches insignifiantes deviennent une fonction. L'oubli de soi, on s'en doute, ne mène pas à rien. C'est dans la logique du dévouement.

Il me parait légitime d'anticiper une telle fiction. Le bureau, nous le savons, d'abord constitué en tant que médiation et représentation tend à devenir ensuite principe d'organisation. Enfin, il a toutes les chances de devenir principe d'aliénation. Car faut-il le rappeler, le bureau a partie liée avec le pouvoir, les délégués, la délégation de pouvoir et donc la dépossession et le détournement ${ }^{16}$; mais aussi avec les stratégies de placement sacerdotal, d'investissement inconscient ou de calcul cynique.

3) Il faut tout de même rendre justice à l'état présent du groupe et de son économie (non utilitariste). C'est une poche d'air dans une mer d'intérêts matériels déchaînés. Poche certes poreuse, mais l'air y est encore respirable. L'économie du groupe est archaïque aux yeux d'un certain nombre d'agents. Pour ces institutionnels, "Corps et Culture » est sans intérêt : on y échange des symboles; sa loi fondamentale est la dénégation de l'économique et le rejet du pouvoir temporel. En somme, le monde de la Faculté des sports à l'envers. C'est donc une bonne chose que "Corps et Culture » soit une entreprise économique pas comme les autres. En disant cela, il n'est pas question de se féliciter. La vraie question demeure la suivante : un laboratoire désintéressé est-il possible ${ }^{17}$ ? 


\section{NOTES}

1. Pour une critique de l'ultra-libéralisme, $\mathrm{Cf}$. les analyses de journalistes et d'universitaires dans Manières de voir 23, Le Monde diplomatique, août 1994 et 35, septembre 1997, respectivement titrés Le modèle Français en Question et Offensives du Mouvement Social. Par ailleurs, le me permets d'attirer l'attention sur les commentaires incisifs de Pierre Bourdieu des déclarations du président de Io Bundesbank, M. Hans Tietmeyer, Surtout son analyse de cette pensée néo-libérale, " aujourd'hui dans la plupart des cerveaux des hommes politiques (de gauche comme de droite), des journalistes et des essayistes vaguement frottés d'économie » (Libération, 23. 10. 1996) et des groupes et des organisations qui la produisent, la répande jusqu'à en faire un stock de savoirs qui s'impose en toute méconnaissance.

2. Cf. par exemple, Ramonet I. (1997) Géopolitique du chaos, Paris, Galilée.

3. Chesneaux 1. (1989). Modernité-Monde, Paris, la Découverte, p. 58.

4. lbid, p. 82.

5. Ibid. pp. 73-74.

6. L'expression est de Halimi S. (1997) Les nouveaux chiens de garde, Paris, Liber-Raisons D'agir. Ce livre est une critique de Io complicité entre les journalistes et les intellectuels-experts-consultants, toujours les mêmes, invités pour donner leur avis sur toutes choses. Ce microcosme s'est approprié L'écran et travaille à imposer l'économie à Io société. On trouvera aussi quelques pages édifiantes concernant le traitement médiatique du mouvement de novembre décembre 1995. A lu lecture des faits d'écran quotidiennement aveuglants, on comprend Io dénonciation de S. Halimi (lui-même journaliste) de la servilité des journalistes à l'égard des pouvoirs et des conseillersexperts à l'aise dans les studios de télévision et prêts à donner ou peuple des leçons de sagesse ou nom de le rationalité économique. Dans un autre registre rhétorique mais tout aussi critique, se reporter à Bourdieu P. (1996) Sur la télévision, suivi de L'emprise du journalisme. Paris, Liber éditions.

7. Pour une analyse des figures de l'engagement (Sartre, Foucault, Bourdieu), Cf. Mauger G. (1995) L'engagement sociologique, Critique. Pierre Bourdieu. N' 579/580, p. 674-696.

8. Sur la circulation instaurée en tant qu'ordre invisible qui est une mise en ordre de la masse des citoyens, on peut se reporter à Virilio P. (1977) Vitesse et politique, Paris, Galilée. L'auteur qui ne l'oublions pas est un urbaniste a fuit des observations très éclairantes sur notre rapport à l'espace et au temps dans les villes modernes et sur l'usage des nouvelles technologies. je ne citerai que Io transformation de notre relation à L'outre à Io fois proche et lointain du fait du passage de la révolution des transports à lu révolution des transmissions et le danger qui guette lu démocratie dans un espacetemps de l'instantanéité et de la télé action. Se reporter par exemple à Cybermonde la politique du pire, Paris, Textuel, 1996.

9. Dans les premières réunions-affrontements le discours de l'origine avait statut de symptôme. Le « vrai » père fondateur qui n'est plus eux commandes depuis plusieurs années, se voyait ravir la position par un prétendant à la fondation qui n'est pas l'actuel Doyen. Outre qu'il y avait du meurtre dans l'air, j'enjeu se traduisait en affrontement entre deux figures (pouvoir/autorité) ; deux principes de hiérarchisation et leur mode de relation avec le champ bureaucratique (autonomie/hétéronomie); deux types 
d'accumulation (celui qui a hérité/celui qui hérite l'aspiration à posséder). Foute de place, je ne tiens pas à rentrer dans plus de détails. Livrer des noms ne sera pas plus éclairant.

10. On trouvera dans Farrugia F. (1993). La crise du lien social, Paris, L'Harmattan, un exposé succinct et clair des concepts à l'oeuvre chez Durkheim et Tönnies, (respectivement, solidarité organique/ solidarité mécanique ; communauté/société) qui sont constitutifs de ce qu'on désigne aujourd'hui par lien social dans une littérature à la mode où l'on découvre sons relâche la perte du lien et la manière de le réinstaurer, Dans Nisbet Robert A. (1993) La tradition sociologique, Paris, Quadrige/PUF, un chapitre est consacré ou concept de communauté. En outre, sur la sociabilité syncrétique et la sociabilité par interaction, cf. Bleger J., (1970) le groupe comme institution et le groupe dans les institutions, in R. Kaës et col. (1988) L'institution et les institutions. Etudes psychanalytiques, Paris, Dunod, coll. Inconscient et culture, pp. 47-61.

11. Sur la mémoire en tant que forme de pathologie institutionnelle, cf. Kaës R. et col (1996) Souffrance et psychopathologie des liens institutionnels, Paris, Dunod, coll. Inconscient et culture, pp. 105-119.

12. Dernière nouvelle ces petits monstres institutionnels, soudés par une idéologie micro-groupale, sont en proie, de nouveau, à un accès de fièvre Toujours sur je même thème délirant (« nous ne sommes plus rien »; « on dit de nous "les petits profs" »; etc. .), ils s'érigent, comme par déni de leur castration institutionnelle, contre tous les autres (I'extérieur à leur groupe) avec des phrases stéréotypées comparables à celles du milieu intellectuel et culturel de " nique I'école nique les profs » mais traduisant un jargon spécifique à leur ressentiment : « tous des cons ! » «X est un con »; « Ies théoriciens, c'est des cons »; « la théorie c'est de la connerie ». Très riche tout ça. Plus encore, ils " foutent » dehors, d'un module de théorie des pratiques, toute personne qui sache lire et écrire afin d'avoir les mains libres et convertir leurs contenus d'enseignement en argent (heures complémentaires) et aussi veiller à transmettre aux étudiants leur culture d'hommes de terrain (niveau universitaire = gazon).

13. Kaës R, et col. (1996) traite du travail de la mort dans les institutions pp. 121-159.

14. Nisbet Robert A., op. cit, p. 185.

15. Caillé A. (1994) Don, intérêt et désintéressement, Paris, La Découverte/MAUSS, pp. 232-296. On peut aussi se reporter avec profit à la conférence prononcée en 1993 par Godbout Jacques T. in 1996 Le langage du don, Montréal, Editions Fides.

16. Bourdieu P. (1981) Choses dites, Paris, Minuit, pp. 185-202.

17. Une analyse et des éléments de réponses à la question épistémologique mais également éthique de "l'intérêt au désintéressement » dans Bourdieu P (1994) Raisons pratiques. Sur la théorie de l'action, Paris, Seuil, pp. 149-167.

\section{AUTEUR}

\section{MAHMOUD MILIANI}

Faculté des Sciences du Sport et de l'Education Physique. Université Montpellier 1. Equipe

«Corps et Culture » 